\title{
Effect of anticonvulsants on plasma testosterone and sex hormone binding globulin levels
}

\author{
J . M . B A R R A G R Y, H L. J . MAK I N, D. J . H . T R A F F O R D, \\ A N D D. F. S C O T T \\ From the Departments of Metabolism and Endocrinology and Chemical Pathology, \\ and the Section of Neurological Sciences, The London Hospital and Medical College, London
}

SUMMARY Plasma sex hormone binding globulin (SHBG) and testosterone levels were measured in 29 patients with epilepsy (16 men and 13 women), most of them on chronic therapy with anticonvulsant drugs. Sex hormone binding globulin concentrations were increased in both sexes and testosterone levels in male patients. It is postulated that anticonvulsants may induce hepatic synthesis of SHBG.

Certain anticonvulsant compounds, notably phenobarbitone and phenytoin, influence many aspects of hormone metabolism. Thus alterations have been described in cortisol secretion, pituitary release of gonadotrophins, corticotrophin, and antidiuretic hormone. Moreover, testosterone and oestradiol catabolism may be increased, presumably by hepatic microsomal hydroxylases (Woodbury et al., 1972). The finding of a grossly elevated level of testosterone in the plasma of an impotent patient with epilepsy on anticonvulsant therapy, prompted an investigation into the effect of such treatment on concentrations of plasma testosterone and SHBG.

\section{Methods}

Sixteen male patients with epilepsy (mean age 34 years) and 13 female epileptics (mean age 31 years) were studied. They suffered mainly from drugresistant temporal lobe epilepsy. Anticonvulsant therapy included one or more of the following compounds: phenobarbitone, phenytoin, carbamazepine, and sodium valproate. In most instances the duration of drug treatment was between three and 10 years although two patients were studied within three months of starting therapy. None was taking any other compound nor was there any evidence of thyroid or hepatic disease, both of which may elevate SHBG concentrations (Anderson, 1974). Blood was drawn for estimation of

Address for reprint requests: Dr D. F. Scott, EEG Department, The London Hospital, Whitechapel, London E1 1 BB.

Accepted 22 May 1978 testosterone, cortisol, total thyroxine, $\mathrm{T} 3$ resin binding, and vitamin D binding globulin (VDBG). Plasma SHBG capacity was measured using the method of Rudd et al. (1974). The results were compared with those obtained from a group of 14 male and 13 female age-matched normal control subjects, members of the hospital staff, none of whom were on a contraceptive pill or receiving other medication.

\section{Results (Table)}

The mean plasma testosterone concentration in male patients with epilepsy receiving anticonvulsant drugs was significantly greater than that of the male unmedicated control group $(P<0.02)$. Among the females with seizures, all of whom were on anticonvulsant drugs, plasma testosterone values were similar to those of the female control group, none of whom were receiving medication $(P>0.05)$. The plasma SHBG concentration in males with epilepsy was greater than that for the male control group $(P<0.01)$. A similar trend was observed in the female patients with seizures when compared with the healthy female subjects $(\mathrm{P}<0.05)$. Plasma cortisol, thyroxine, T3 resin

Table Plasma levels of testosterone and SHBG. Mean and standard deviation. Control values in parentheses

\begin{tabular}{lcc}
\hline Hormone & Men & Women \\
\hline Testosterone & $30 \quad \pm 17.1$ & $2.5 \pm 1.4$ \\
(nmol/l) & $(16 \pm 3.7)$ & $(1.5 \pm 0.8)$ \\
SHBG & $7.32 \pm 1.4$ & $9.19 \pm 1.87$ \\
$\left(10^{-2} \mathrm{~mol} / \mathrm{l}\right)$ & $(5.76 \pm 0.82)$ & $(7.67 \pm 1.10)$ \\
\hline
\end{tabular}


binding, and VDBG were normal in the patients. No correlation was noted between the testosterone and SHBG levels nor was there any relationship between these and the amount of anticonvulsant drug being administered. In all instances where testosterone and SHBG levels were raised, drug therapy included phenobarbitone, phenytoin or carbamazepine. In two patients studied within three months of the start of phenobarbitone and phenytoin therapy normal amounts of SHBG were found.

\section{Discussion}

A previous study has demonstrated an increased plasma concentration of SHBG in females on phenytoin therapy (Victor et al., 1977). In the present series a similar pattern was seen. Further, there was a significant rise in SHBG levels in male patients, associated with an increased plasma concentration of testosterone. Normal values of T3 resin binding, thyroxine, cortisol, and VDBG were found, and these findings are compatible with a selective effect of anticonvulsant drugs on hepatic SHBG synthesis rather than a general enhancement of transport protein synthesis. The results of the present study also suggest that, in addition to phenytoin, long-term administration of phenobarbitone or carbamazepine is also associated with increased plasma concentration of SHBG. In males, this increase will enhance the total plasma testosterone concentration when there is a normal pituitary-testis axis. The production of testosterone in females is not regulated by similar means, and so the finding of normal plasma levels of testosterone is not unexpected.

From a clinical standpoint it is important that these anticonvulsant-induced changes in SHBG concentration be kept in mind when hormonal aspects of sexual function are being investigated in patients on anticonvulsant therapy.

Moreover, these changes in SHBG concentration might cause a disturbance of sexual function. Definite statistical information is lacking about the prevalence of sexual disturbance in epilepsy, and there are reports both of diminished sexual activity and of hypersexuality, especially in temporal lobe epilepsy (Scott, 1978). With one exception, no history of a sexual disorder was elicited in this series. The principal hormonal determinant of sexual performance is the circulating level of free rather than protein-bound hormone, and it seems improbable that the observed changes in plasma SHBG capacity and testosterone concentration by themselves would alter sexual function significantly. Nevertheless, under circumstances of increased protein binding of testosterone and altered pituitary release of gonadotrophins, both of which may be associated with anticonvulsant therapy, a reduction in plasma-free testosterone and therefore sexual function might be anticipated. This suggestion is in keeping with the findings of Christiansen et al. (1977) who reported a high incidence of impotence in male patients with epilepsy, on phenobarbitone and phenytoin, in association with decreased excretion of androgens.

We wish to thank Dr A. Stuart Mason for his help and encouragement.

\section{References}

Anderson, D. C. (1974). Sex-hormone binding globulin. Clinical Endocrinology, 3, 69-96.

Christiansen, P., Deibjer, J., and Lund, M. (1977) Eighth International Symposium on Epilepsy Dublin 1976. Cited in British Journal of Sexuab Medicine, 4, 8.

Rudd, B. T., Duignan, N. M., and London, D. R. (1974). A rapid method for the measurement of sexhormone binding globulin capacity of serum. Clinica Chimica Acta, 55, 165-178.

Scott, D. F. (1978). Sexual functioning in epilepsy. British Journal of Sexual Medicine, 5, 17-18. (Part 2 in press)

Victor, A., Lundberg, P. O., and Johansson, E. D. B. (1977). Induction of sex hormone binding globulin by phenytoin. British Medical Journal, 2, 934-935.

Woodbury, D. M., Penry, J. K., and Schmidt, R. P. (1972). Anti-epileptic Drugs. Raven Press: New York. 